Disclosure of Interest: None declared

DOI: 10.1136/annrheumdis-2017-eular.3647

\section{SAT0015 ANCA-ASSOCIATED VASCULITIS- AND SYSTEMIC LUPUS ERYTHEMATOSUS-INDUCED NEUTROPHIL EXTRACELLULAR TRAPS HAVE INTRINSICALLY DIFFERENT FEATURES}

L.S. Van Dam ${ }^{1}$, T. Kraaij ${ }^{1}$, S.W. Kamerling ${ }^{1}$, M.C. Avramut ${ }^{2}$, C.R. Jost ${ }^{2}$, A.J. Koster ${ }^{2}$, H.U. Scherer ${ }^{3}$, C.D. Pusey ${ }^{4}$, A.J. Rabelink ${ }^{1}$, C. van Kooten ${ }^{1}$, Y.K. Teng ${ }^{1} .{ }^{1}$ Nephrology; ${ }^{2}$ Molecular Cell Biology; ${ }^{3}$ Rheumatology, LUMC, Leiden, Netherlands; ${ }^{4}$ Renal and Vascular Inflammation, Imperial College, London, United Kingdom

Background: Neutrophil extracellular traps (NETs) are immunogenic, extracellular DNA structures that harness important autoantigens to be recognized by the adaptive immune system. NETs are thought to play a pivotal role in the pathogenesis of many systemic autoimmune diseases including ANCA-associated vasculitis (AAV) and systemic lupus erytematosus (SLE). However it is still unclear how and if NETs can act as a common pathway in the pathophysiology of these clinically divergent autoimmune diseases.

Objectives: To investigate the characteristics of NETs induced by sera of AAV and SLE patients.

Methods: The present study involved 101 AAV patients, 59 SLE patients and 10 healthy controls. Healthy neutrophils were stimulated with $10 \%$ serum of these patients to induce NETs. Quantity of NET induction was measured by a novel, highly-sensitive NET quantification assay using 3D-confocal laser scanning microscopy ${ }^{1}$. Qualitative characteristics of NETs were investigated by immunofluorescence microscopy that detected co-localisation of several established autoantigens and NET-markers on AAV- and SLE-induced NETs, including citrullinated histon-3 (CitH3), neutrophil elastase (NE), high mobility group box-1 (HMGB1), myeloperoxidase (MPO) and proteinase-3 (PR3). Additionally, the morphology and kinetics of AAV- and SLE-induced NETosis were visualized by live imaging and electron microscopy.

Results: Quantifying ex vivo NET induction demonstrated that AAV sera induced significant more NETs (median [Q1 - Q3]: 20.74 [9.56 - 74.14]), compared to SLE sera $(5.02$ [1.88 - 14.33]). Also qualitatively, NETs induced by AAV or SLE sera were distinct. In both cases, NETs showed co-localisation of MPO and PR3 with extracellular DNA. However, AAV-induced NETs had significantly higher Cith3 expression than SLE-induced NETs. Interestingly, the opposite was observed for other markers as HMGB1 was exclusively expressed on SLE-induced NETs and NE was also higher expressed on SLE-induced NETs compared to AAV-induced NETs. Intriguingly, the distinction between AAV and SLE NETs was further corroborated by live imaging demonstrating differences in morphology and chronology of NET induction: in SLE NET-clusters were induced within the 1st hour while in AAV non-clustered NETs composed of long, thin DNA-fibres were induced in 2-4 hours through lytic expulsion.

Conclusions: The present study demonstrates that NET induction in AAV and SLE results in quantitative and qualitative distinct NETs indicating that NET formation in AAV and SLE is likely based on intrinsically different processes. These data increase our understanding of the pathophysiologic relevance of NETs and how they could be considered as a common pathway underpinning different autoimmune diseases.

References:

[1] Kraaij T, et al, A novel method for high-throughput detection and quantification of neutrophil extracellular traps reveals ROS-independent NET release with immune complexes, Autoimmun Rev (2016).

Disclosure of Interest: None declared

DOI: 10.1136/annrheumdis-2017-eular.3804

\section{SAT0016 A NEW SUBSET OF NK CELLS, WITH ENHANCED CYTOTOXIC FUNCTION, IS INCREASED IN SYSTEMIC LUPUS ERYTHEMATOSUS PATIENTS}

D.D.J. Cruz González ${ }^{1}$, L. Baranda-Cándido ${ }^{2}$, C. Abud-Mendoza ${ }^{2}$, J. Alcocer-Varela ${ }^{3}$, R. González-Amaro ${ }^{1}$, D. Gómez-Martin ${ }^{3}$,

A.E. Monsiváis-Urenda ${ }^{1}$. ${ }^{1}$ Medicina Translacional y Molecular. Centro de Investigación en Salud y Biomedicina; ${ }^{2}$ Departamento de Reumatología. Hospital Central "Ignacio Morones Prieto", Facultad de Medicina. Universidad Autónoma de San Luis Potosí, San Luis Potosí; ${ }^{3}$ Departamento de Reumatología e Inmunología, Instituto Nacional de Ciencias Médicas y Nutrición Salvador Zubirán, Ciudad de México, Mexico

Background: Natural killer cells (NK cells) are granular lymphocytes that belong to innate immunity, its major function is the lysis of virus-infected or tumoral cells. These functions are regulated by activating (NKG2D, NKp46, NKP30, NKG2C, CD161) and inhibitory receptors like KIRs and NKG2A. The role of NK cells in autoimmunity is poorly understood; it is well known that in systemic lupus erythematosus (SLE) patients there are decreased levels of NK cells. NK cells can modulate the adaptive immunity through its interaction with dendritic cells (DCs); the activation or killing of DC by NK cells depends of the ratio NK:DC. However, the interactions NK:DC in SLE have not been well studied. A new subset of NK cells with DCs characteristics has been reported in a model of lupus mice; however, the possible increment of NK cells with DC like phenotype in SLE has not been reported.
Objectives: To analyze the phenotype of circulating NK cells as well as its function in SLE patients.

Methods: Sixty SLE patients and fifty-five controls were included in this study. Diagnosis was made according to ACR criteria. Activity of disease was measured by SLEDAI index. The expression of NKG2A, ILT2, NKG2D, NKG2C, NKp30, NKp46, CD161, CD134, CD80, CD86, HLA-DR, CD11c+ was evaluated in NK cells (CD3-CD56+) from peripheral mononuclear cells. NK cell function was assessed by the percentage of monocyte-derived DC lysis by NK cells.

Results: Diminished levels of circulating NK cells were found in SLE patients $(p=0.0439)$ compared to healthy subjects. NK cells from SLE showed higher levels of the inhibitory receptor ILT2+( $=0.0024)$, and the costimulatory molecules CD86+ $(p=0.0136)$ and $C D 134+(p=0.0238)$; in addition, SLE patients displayed a higher expression of MHC-class II molecule, HLA-DR+ $(p<0.0001)$. Interestingly, higher levels of atypical NK cells CD11c+HLA-DR+ $(p=0.0075)$ were found in SLE patients compared with healthy subjects. Furthermore, we found that SLE patients showed a significate increased level of monocyte derived-DC lysis by NK cells.

Conclusions: In this study, we show for the first time that NK cells in SLE have an altered phenotype, expressing receptors, which are characteristic of dendritic cells (CD134, CD86 and HLA-DR). The expression of these receptors may provide NK cells with the ability to activate $T$ cells, which together with their higher capacity to lyse immature or tolerogenic DCs could contribute to SLE pathogenesis. It is known that NK cells could have a dual role in autoimmune diseases, here we propose that the lysis of DC mediated by NK cells could be important to modulate the disease activity in SLE patients. Even more, in this report we identify a new subset of NK cells, CD11C+ HLA-DR+, reported previously in a mouse lupus model. It is essential to highlight that these NK cells with DC-like phenotype could be crucial for the development of SLE.

References:

[1] Caligiuri MA. Bone 2008; 112: 461

[2] Voynova EN, et al. J Immunol 2015; 194: 1503.

[3] Aranami T, et al. J Immunol 2006;177: 5659.

Disclosure of Interest: None declared

DOI: 10.1136/annrheumdis-2017-eular.3565

\section{SATURDAY, 17 JUNE 2017 Adaptive immunity ( $T$ cells and $B$ cells) in rheumatic} diseases

\section{SAT0017 INVESTIGATING NOVEL AUTOANTIBODIES IN PATIENTS WITH GRANULOMATOSIS WITH POLYANGIITIS}

M.C. Baker, N. Lingampalli, W. Robinson. Rheumatology, Stanford University, Palo Alto, United States

Background: Granulomatosis with polyangiitis (GPA) is a disease characterized by inflammation in small blood vessels, leading to significant organ damage and ultimately great morbidity and mortality. The presence of anti-neutrophil cytoplasmic antibodies (ANCAs) is a hallmark of the disease, and thought to directly play a role in pathogenesis by activating primed neutrophils. The two major antigens that ANCAs are believed to recognize are myeloperoxidase (MPO) and proteinase 3 (PR3). Although anti-MPO antibodies are directly pathogenic when transferred or induced in animal models, the evidence is less clear for PR3. Clinically, it is debated whether levels of anti-PR3 correlate with disease activity. Therefore, we believe that anti-PR3 antibodies are not the primary drivers of disease in patients with PR3+ ANCA-vasculitis (i.e. GPA), and that novel autoantibodies may play a role in disease pathogenesis.

Objectives: The objective of this work is to identify the human autoantigen targets of novel autoantibodies identified in GPA, and to test their association with disease. The goal is to discover novel autoantigens that will ultimately lead to new diagnostics and therapeutics.

Methods: Cell-barcode-enabled antibody repertoire sequencing was performed on blood plasmablasts (antibody-producing cells formed during an immune response) from five PR3+ ANCA-vasculitis patients with GPA treated with rituximab in the RAVE trial. Phylogenetic trees were bioinformatically created in order to identify clonal families of plasmablasts. Antibodies representing clonal families of plasmablasts were recombinantly expressed and are being tested using human protein arrays and ELISAs to determine their antigen specificity. Results: All five PR3+ ANCA-vasculitis patients sequenced from the RAVE trial achieved complete remission but subsequently flared. Plasmablasts were isolated at the baseline flare, at remission and at the post-rituximab flare. Phylograms of the antibody repertoires revealed clonal families of affinity-matured antibodies that share heavy and light chain VJ usage. A total of 24 representative antibodies were selected for recombinant expression, including representative antibodies derived from clonal families: (1) shared across patients at baseline flare and/or post-rituximab flare $(n=5)$, (2) present at baseline flare and post-rituximab flare $(n=7)$ [persistent clone or same clone came back], (3) present at baseline flare or post-rituximab flare $(n=6),(4)$ present in remission and post-rituximab flare $(n=5)$ [achieves remission despite clone presence], (5) present in remission (but not flare) $(n=1)$ [patient in remission despite clone presence]. None of the 24 antibodies bind PR3 in an ELISA and thus these antibodies do not represent 
ANCAs. Antigen discovery to determine the targets of these recombinant plasmablast antibodies is ongoing.

Conclusions: Plasmablasts from patients with GPA in the RAVE trial were sequenced and antibody repertoires were generated. Clonal families of plasmablasts, including those expressing antibodies possessing shared CDR sequences across multiple patients, were identified and recombinantly expressed. None of the 24 antibodies bound to PR3, suggesting these are not ANCAs and are potentially novel autoantibodies. Identification of the antigen targets of these antibodies is ongoing.

Acknowledgements: The Immune Tolerance Network

Training Program in Adult and Pediatric Rheumatology 2T32AR050942-11

Disclosure of Interest: None declared

DOI: 10.1136/annrheumdis-2017-eular.3261

\section{SAT0018 EFFECTS OF ANTI-TNF ALPHA THERAPY ON B CELLS IN RHEUMATOID ARTHRITIS (RA) PATIENTS}

O. Pala $^{1}$, A. Diaz ${ }^{2}$, B. Aubourg ${ }^{1}$, E. Greidinger ${ }^{1}$, B.B. Blomberg ${ }^{2}$, D. Frasca ${ }^{2}$ ${ }^{1}$ Rheumatology; ${ }^{2}$ Microbiology\&Immunology, University of Miami, Miami, United States

Background: Our laboratory has previously characterized defects in humoral B cell responses of aged mice and humans. These defects include: the reduction in activation-induced cytidine deaminase (AID), required for the generation of optimal antibody responses, and the reduction in the percentage/number of the subset of switched memory B cells (1). AID and switched memory B cells have been proposed to be effective predictive biomarkers of vaccine responses (2). Moreover, we have shown that aging is characterized by increased systemic inflammation which induces intrinsic B cell inflammation, measured by intracellular (ic) TNF- $\alpha$, and this significantly decreases the capacity of the same B cells to make protective antibodies in response to vaccination (3). Other marker of $B$ cell intrinsic inflammation is micro-RNA (miR) expression, particularly miR-16 and miR-155, which is increased in elderly $B$ cells and negatively correlated with $B$ cell function (4)

Objectives: Our goal for this study was to evaluate B cell phenotype and function in RA patients treated Methotrexate (MTX), alone or together with anti-TNF- $\alpha$. We hypothesized that patients treated with anti-TNF- $\alpha$ will show improved B cell function due to reduction in icTNF- $\alpha$.

Methods: We recruited 9 RA patients, 5 patients on MTX and 4 on MTX/anti-TNF$\alpha$. We measured the relevant $B$ cell subsets in blood (Naïve, switched memory, IgM memory and late memory) by flow cytometry. Staining was performed with antibodies specific for CD19, CD27 and IgD. In addition, we isolated blood B cells using magnetic beads, and measured the expression of miR-16 and miR-155 on blood B cells by qPCR.

Results: Preliminary data showed that the percentages of switched memory (IgD-CD27+) B cells are significantly higher $(\mathrm{p}<0.003)$ in patients undergoing MTX/anti-TNF- $\alpha$ therapy. We also observed a significant decrease in naïve B cell percentages $(p<0.028)$. Preliminary results also showed a decrease in the mRNA expression of both miR-16 and miR-155 in patients on combination therapy compared to MTX alone.

Conclusions: These results support the hypothesis that therapy with anti-TNF- $\alpha$ is beneficial for improving $B$ cell function in RA patients, as compared to MTX therapy alone. Future experiments will seek to evaluate intrinsic B cell TNF levels in these patients and correlate them with measurements of $B$ cell function. Treatment with anti-TNF- $\alpha$ may be able to block the excessive amounts of systemic TNF- $\alpha$ and in turn B cell intrinsic TNF- $\alpha$ which could improve the antibody responses and the risk of infections in RA patients undergoing therapy. References:

[1] Frasca, D., and B. B. Blomberg. 2013. Age effects on mouse and human B cells. Immunol Res. 57(1-3): 354-360.

[2] Frasca, D., A. Diaz, M. Romero, M. Phillips, N. V. Mendez, A. M. Landin, and B. B. Blomberg. 2012. Unique biomarkers for B-cell function predict the serum response to pandemic H1N1 influenza vaccine. Int Immunol. 24(3): 175-182.

[3] Frasca, D., M. Romero, A. Diaz, S. Alter-Wolf, M. Ratliff, A. M. Landin, R. L. Riley, and B. B. Blomberg. 2012. A Molecular Mechanism for TNF-alphaMediated Downregulation of B Cell Responses. J Immunol 188: 279-286.

[4] Frasca, D., A. Diaz, M. Romero, F. Ferraci, and B. B. Blomberg. 2015. MicroRNAs miR-155 and miR-16 Decrease AID and E47 in B Cells from Elderly Individuals. J. Immunol.195 (5): 2134-2140.

Disclosure of Interest: None declared

DOI: 10.1136/annrheumdis-2017-eular.4331

\section{SAT0019 ESTROGEN INFLUENCES THE SIALYLATION PROFILE AND INFLAMMATORY PROPERTIES OF ANTIBODIES - A POTENTIAL EXPLANATION FOR THE SEX DIFFERENCES AND INCREASED RISK FOR RA IN POSTMENOPAUSAL WOMEN}

C. Engdahl $^{1,2}$, J. Raufer ${ }^{1}$, U. Harre ${ }^{1}$, A. Bondt ${ }^{3}$, R. Pfeifle ${ }^{1,4}$, G. Krönke ${ }^{4,5}$, H.U. Scherer ${ }^{6}$, H. Forsblad ${ }^{2,7}$, G. Schett ${ }^{1} .{ }^{1}$ Department of Internal Medicin 3, Clinical Immunolgy, Erlangen, Germany; ${ }^{2}$ Department of Rhemtology and Inflammation reserch, CBAR, Institue of Medicine, Gothenburg, Sweden; ${ }^{3}$ Department of Rheumatology, Lediden University Medical Center, Leiden,
Netherlands: ${ }^{4}$ Nikolaus-Fiebiger Center for Molecular Medicine, FAU Erlangen-Nuremberg, Erlangen; ${ }^{5}$ Department of Internal Medicin 3, Clinical Immunolgy, Gothenburg, Germany; ${ }^{6}$ Department of Rheumatology, Lediden University Medical Center, Leiden, Netherlands; ${ }^{7}$ Department of Public Health and Clinical Medicine, Rheumatology, Umeå University, Umeå, Sweden

Background: Rheumatoid arthritis preferentially affects women. RA has its peak over 50 years coincidencing with the decrease in sex hormones in menopause. Recently, the transition from asymptomatic autoimmunity to RA has been shown to essentially depend on the glycosylation status of antibodies affecting the binding affinity to Fc gamma receptors ${ }^{1}$. Hence a decrease in the sialylation of antibodies resulting from a decrease in the activity of the sialylation enzyme $\beta$-galactoside $\alpha 2,6$-sialylltransferase (St6Gal1) was shown to trigger the onset of RA.

Objectives: To test whether estrogen influences the glycosylation status of antibodies and St6Gal1 expression explaining why postmenopausal women are particularly prone to develop RA

Methods: In the experimental part we tested the influence of estrogen on antibody glycosylation and St6Gal1 expression. Ovariectomized mice, which were either left without estrogen supplementation or were supplemented with estrogen (hormone replacement), were immunized with ovalbumin (OVA) to induce antibody production. Immunoglobulin G ( $\mathrm{lgG}$ ) levels were analyzed by ELISA and the glycosylation of the Fc-part of total and OVA-specific IgG was determined by lectin ELISA and MALDI-TOF, respectively. St6Gal1 expression in plasma cells was determined by RT-PCR and FACS. Inhuman part we measured the effects of estrogen treatment on autoantibody levels and IgG glycosylation in a cohort of postmenopausal RA patients over 2 years ${ }^{2}$.

Results: Ovariectomy and loss of estrogens was associated with a lower sialylation of OVA-specific IgG. Conversely estrogen treatment significantly increased the sialylation level of newly formed OVA-specific and totals IgG as well as enhanced the expression of St6Gal1 enzyme in plasma cells suggesting a shift towards an anti-inflammatory pattern of IgG. These results were confirmed with estrogen treated postmenopausal RA patients showing that hormone replacement therapy significantly increased antibody glycosylation, while in a control RA population not exposed to estrogens no such increase in sialylation of IgG was found. Estrogens however, did not influence the CCP autoantibody levels. Conclusions: These findings indicate that estrogen regulates St6Ga1 and increases the glycosylation of IgG. Lack of estrogen decreases lgG glycosylation and results in pro-inflammatory properties of $\lg \mathrm{G}$ which may explain the increase prevalence of RA in postmenopausal women.

\section{References:}

[1] Pfeifle et al. Regulation of autoantibody activity by the IL-23- $T_{H} 17$ axis determines the onset of autoimmune disease. Nat Immunol. 2017 Jan;18(1):104113.

[2] D'Elia HF, et al. Influence of hormone replacement therapy on disease progression and bone mineral density in rheumatoid arthritis. J Rheumatol. 2003 Jul;30(7):1456-63.

Disclosure of Interest: None declared

DOI: 10.1136/annrheumdis-2017-eular.6287

\section{SAT0020 THE CITRULLINOME IN TISSUE AND BIOFLUIDS OF HUMAN AND MOUSE ORIGIN}

T.B.G. Poulsen ${ }^{1}$, D. Damgaard ${ }^{2}$, T.B. Bennike ${ }^{1}$, M.K. Meyer ${ }^{3}$, K.J. Elbæk ${ }^{1}$, V. Andersen ${ }^{4}$, S. Birkelund ${ }^{1}$, C.H. Nielsen ${ }^{2}$, A. Stensballe ${ }^{1} .{ }^{1}$ Department of Health Science and Technology, Aalborg University, Aalborg; ${ }^{2}$ Center for Rheumatology and Spine Diseases, Copenhagen University Hospital, Rigshospitalet, Institute for Inflammation Research, Rigshospitalet, Institute for Inflammation Research, Copenhagen: ${ }^{3}$ Department of Rheumatology, North Denmark Regional Hospital, Hjørring; ${ }^{4}$ IRS-Center Sonderjylland, Hospital of Southern Jutland, Aabenraa, Denmark

Background: Protein citrullination is fundamental to several essential processes in apoptosis and antimicrobial defense, however, also linked to multiple pathogenic endpoints. This post-translational modification (PTM), by conversion of arginine to citrulline residues, is mediated by peptidylarginine deiminase (PAD) enzymes found in specific cells and tissues. In polymorphonuclear cells (PMNs) these enzymes enable NETosis, a specialized form of programmed necrosis and the formation of NETs (neutrophil extracellular traps). Also, these enzymes are expressed in the synovium of patients with rheumatoid arthritis (RA) thereby triggering the production of autoantibodies against citrullinated proteins (ACPAs). Objectives: Our objective was to optimize methodology for characterization of this PTM and determine the citrullinome in tissue and biofluids of human and mouse origin in clinical relation to rheumatoid arthritis (RA), osteoarthritis (OA), Spondyloarthritis (SpA) as well as presence of ACAPs and NETs

Methods: Synovial fluid (SF) and plasma was collected from patients diagnosed with RA, OA, SpA $(n=120)$. Inflammation levels patients were characterized with plasma C-reactive protein (CRP), and circulating anti-CCP levels as well as 10 most relevant proinflammatory cytokines. Intestinal tissue (colon mucosa) from RA patients $(n=10)$ and joint lysate from collagen-induced arthritis mouse model $(n=24)$. All samples were analyzed by citrulline specific sample preparation and high-end mass spectrometric analysis [1,2]. Follow-up studies were performed by multiple techniques including confocal microscopy and cell-free DNA measurement. 